Supplementary Table 1: Summary Statistics for 98 quantitative traits

\section{Age Band}

\# individual $\uparrow$

Blood Test Analyses

RBC

$\mathrm{MCV}$ (mean RBC vol)

$\mathrm{MCH}$ (mean RBC Hb)

WBC

NE (neutrophils)

LY (lymphocytes)

MO (monocytes)

EO (eosinophils)

BA (basophils)

PLT (platelets)

$\mathrm{HbF}$

$\mathrm{HbA} 2$

HbA1C

G6PD

SERUM GLUCOSE

SERUM INSULIN

BUN

SERUM CREATININE

ALT, ala aminotransferase

AST, asp aminotransferase $U / L$

Gammagt, $\gamma$-glu-transferase $\quad U / L$

FIBRINOGEN

CHOLESTEROL

HDL

LDL

TRIGLYCERIDES

IRON

TRANSFERRIN

BILIRUBIN, fractionated

BILIRUBIN, total

URIC ACID

SODIUM

POTASSIUM

ESR, erythrocyte sed. rate

CRP, C-reactive protein

TSH, thyroid stim. hormone microU/L

PSA

\section{$\frac{\text { Units }}{10^{6} / \mathrm{uL}}$}

$\mathrm{g} / \mathrm{dL}$

$\mathrm{pg}$

$10^{3} / \mathrm{uL}$

$\%$

$\%$

$\%$

$10^{3} / \mathrm{uL}$

$\%$

$\%$

$\mathrm{UI} / \mathrm{dL}$

$\mathrm{mg} / \mathrm{dL}$

$\mathrm{mg} / \mathrm{dL}$

$U / L$

$\mathrm{mg} / \mathrm{dL}$

$\mathrm{mg} / \mathrm{dL}$

$\mathrm{mg} / \mathrm{dL}$

$\mathrm{mg} / \mathrm{dL}$

$\mathrm{mg} / \mathrm{dL}$

$\mathrm{mg} / \mathrm{dL}$

$\mathrm{mg} / \mathrm{dL}$

$\mathrm{mg} / \mathrm{dL}$

$\mathrm{mEq} / \mathrm{L}$

$\mathrm{mEq} / \mathrm{L}$

$\mathrm{mm} / \mathrm{h}$

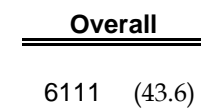

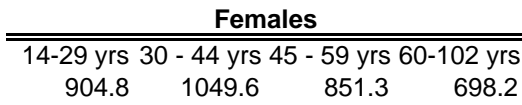

$\begin{array}{cc}904.8 & 1049.6\end{array}$ $\mathrm{mg} / \mathrm{dL}$

microg/dL
$611.7 \quad 564.2$

\subsection{7}

$86.5 \quad(9.3)$

$6.7 \quad(1.7)$

$56.6 \quad(8.7)$

$5.9 \quad(23)$

$2.6 \quad(1.8)$

$0.4 \quad(0.3)$

$243.0 \quad(60.0)$

$0.4 \quad(0.7)$

$3.1 \quad(1.0)$

$5.4 \quad(0.8)$

$\begin{array}{rr}1.2 & (0.4) \\ 90.1 & (23.7)\end{array}$

$\begin{array}{rr}9.5 & (23.7) \\ & (8.1)\end{array}$

$35.0 \quad(10.4)$

\begin{tabular}{ll}
$0.8 \quad(0.2)$ \\
\hline
\end{tabular}

$25.0 \quad(23.1)$

$21.6 \quad(14.6)$

$330.0 \quad$ (67.4)

$\begin{array}{rr}64.1 \quad(14.9) \\ 127.0 & (35.4)\end{array}$

$127.0 \quad(35.4)$

$88.1 \quad$ (68.3)

$87.7 \quad(34.8)$

$307.0 \quad(62.9)$

$\begin{array}{ll}0.1 & (0.1) \\ 0.7 & (0.4)\end{array}$

$4.3 \quad(1.5)$

$143.0 \quad(3.6)$

$4.4 \quad(0.4)$

$\begin{array}{rr}10.6 & (8.2) \\ 0.7 & (1.1)\end{array}$

$1.9 \quad(26)$

$0.8 \quad(1.1)$
14.7
90.2

90.2
30.1

$\begin{array}{rrrr}5.3 & 5.2 & 5.1 & 4.9 \\ 14.9 & 14.9 & 14.9 & 14.7 \\ 85.0 & 86.5 & 88.0 & 90.2 \\ 28.6 & 29.1 & 29.4 & 30.1 \\ 7.1 & 7.0 & 7.1 & 6.9 \\ 54.6 & 56.6 & 56.1 & 57.5 \\ 35.8 & 34.3 & 34.3 & 32.4 \\ 6.6 & 6.0 & 6.3 & 6.7 \\ 2.7 & 2.7 & 2.9 & 3.0 \\ 0.4 & 0.3 & 0.3 & 0.3 \\ 234.0 & 231.0 & 227.0 & 220.0 \\ 0.4 & 0.3 & 0.3 & 0.3 \\ 3.1 & 3.1 & 3.1 & 3.0 \\ 5.2 & 5.3 & 5.8 & 5.9 \\ 1.3 & 1.3 & 1.2 & 1.2 \\ 84.7 & 89.8 & 100.0 & 104.0 \\ 7.4 & 8.1 & 9.8 & 9.0 \\ 34.8 & 37.6 & 37.5 & 40.9 \\ 0.9 & 0.9 & 0.9 & 1.0 \\ 26.6 & 37.2 & 34.1 & 28.6 \\ 22.2 & 24.4 & 25.5 & 25.8 \\ 22.0 & 41.1 & 51.2 & 46.7 \\ 289.0 & 300.0 & 316.0 & 347.0 \\ 175.0 & 213.0 & 226.0 & 220.0 \\ 55.6 & 57.1 & 59.7 & 62.6 \\ 105.0 & 134.0 & 141.0 & 136.0 \\ 72.5 & 111.0 & 127.0 & 106.0 \\ 94.1 & 98.3 & 96.4 & 95.2 \\ 301.0 & 293.0 & 297.0 & 292.0 \\ 0.1 & 0.1 & 0.1 & 0.2 \\ 0.8 & 0.8 & 0.7 & 0.8 \\ 4.8 & 5.1 & 5.4 & 5.7 \\ 142.0 & 143.0 & 143.0 & 143.0 \\ 4.3 & 4.4 & 4.5 & 4.6 \\ 5.8 & 6.4 & 7.1 & 10.9 \\ 0.6 & 0.7 & 0.7 & 0.9 \\ 4.2 & 2.5 & 1.0 & 2.0 \\ 0.5 & 0.6 & 0.7 & 1.4 \\ & & & \end{array}$

Significant Variation

Age Sex

\begin{tabular}{|c|c|c|c|c|c|}
\hline 4.6 & 4.6 & 4.7 & 4.7 & 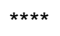 & 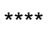 \\
\hline 13.0 & 12.8 & 13.1 & 13.4 & 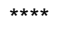 & 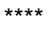 \\
\hline 85.0 & 85.4 & 86.0 & 87.6 & 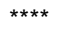 & 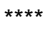 \\
\hline 28.3 & 28.4 & 28.5 & 29.0 & 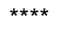 & 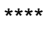 \\
\hline 6.7 & 6.5 & 6.2 & 6.2 & 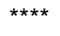 & 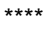 \\
\hline 55.1 & 58.3 & 57.2 & 57.0 & 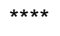 & 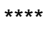 \\
\hline 36.7 & 33.7 & 34.2 & 34.2 & 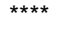 & \\
\hline 5.6 & 5.3 & 5.6 & 5.8 & 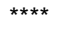 & 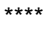 \\
\hline 2.3 & 2.3 & 2.6 & 2.6 & 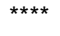 & 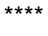 \\
\hline 0.4 & 0.4 & 0.4 & 0.4 & & 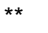 \\
\hline 263.0 & 258.0 & 256.0 & 237.0 & $\star * \star *$ & 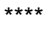 \\
\hline 0.5 & 0.5 & 0.4 & 0.4 & 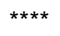 & 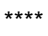 \\
\hline 3.1 & 3.1 & 3.0 & 2.9 & ** & \\
\hline 5.1 & 5.2 & 5.5 & 5.9 & 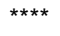 & 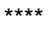 \\
\hline 1.3 & 1.2 & 1.2 & 1.2 & 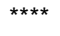 & \\
\hline 79.0 & 82.9 & 90.4 & 100.0 & 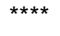 & 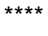 \\
\hline 8.3 & 7.6 & 8.8 & 9.8 & $\star \star \star *$ & \\
\hline 28.4 & 31.5 & 34.8 & 39.9 & 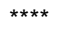 & 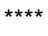 \\
\hline 0.7 & 0.7 & 0.7 & 0.8 & 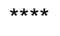 & 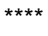 \\
\hline 16.0 & 17.9 & 22.5 & 24.8 & $\star \star * * *$ & 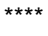 \\
\hline 17.2 & 17.4 & 20.7 & 24.2 & 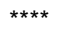 & 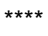 \\
\hline 13.8 & 15.8 & 21.9 & 27.7 & 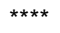 & 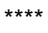 \\
\hline 326.0 & 332.0 & 348.0 & 375.0 & 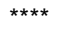 & 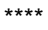 \\
\hline 185.0 & 204.0 & 226.0 & 228.0 & 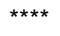 & \\
\hline 66.3 & 68.3 & 69.7 & 69.0 & 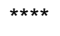 & 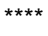 \\
\hline 106.0 & 121.0 & 139.0 & 140.0 & $\star \star * \star *$ & ** \\
\hline 61.8 & 69.9 & 84.8 & 95.4 & 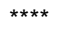 & 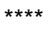 \\
\hline 80.2 & 80.1 & 81.1 & 85.7 & * & 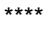 \\
\hline 326.0 & 325.0 & 308.0 & 297.0 & 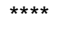 & 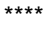 \\
\hline 0.1 & 0.1 & 0.1 & 0.1 & 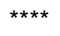 & 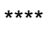 \\
\hline 0.6 & 0.6 & 0.6 & 0.7 & 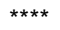 & 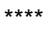 \\
\hline 3.2 & 3.3 & 3.8 & 4.4 & 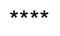 & 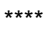 \\
\hline 142.0 & 142.0 & 143.0 & 143.0 & 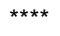 & 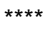 \\
\hline 4.3 & 4.4 & 4.5 & 4.5 & 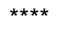 & 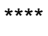 \\
\hline 11.0 & 11.9 & 13.4 & 16.8 & 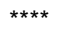 & 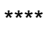 \\
\hline 0.7 & 0.7 & 0.8 & 0.9 & 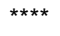 & \\
\hline 1.8 & 1.9 & 1.9 & 1.7 & & \\
\hline
\end{tabular}


Age Band

\# individual

Anthropometric Measures

HEIGHT

WEIGHT

WAIST

$\mathrm{BMI}$, body mass index

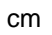

$\mathrm{kg}$

$\mathrm{cm}$

$\mathrm{cm}$

Cardiovascular Function

systolic BP

diastolic BP

HR (heart rate)

$\mathrm{mmHg}$

$\mathrm{mmHg}$

diam_S

beats/min

$\mathrm{mm}$

$\mathrm{mm}$

MT (intimal medial thickness) $\mathrm{mm}$

PWV (pulse wave velocity) $\mathrm{cm} / \mathrm{s}$

\section{Derived variable}

pulse pressure

mean BP

Wall/lumen

Vascular mass

normalized PWV

$\mathrm{mmHg}$

no units

$\mathrm{mg} / \mathrm{mm}$

$\mathrm{cm} / \mathrm{s} / \mathrm{mmHg}$

EKG variables

QTC (QT interval on EKG)

PR (PR interval on EKG) msec

Sonographic variables

PSV (peak systolic velocity) $\mathrm{cm} / \mathrm{s}$

EDV (end diastolic velocity) $\mathrm{cm} / \mathrm{s}$

IP (pulsatility index)

$\mathrm{cm} / \mathrm{s}$
no units

SD_ratio

no units

AT (acceleration time)

ms

vti (integral time velocity)

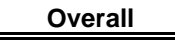

$6111 \quad$ (43.6)

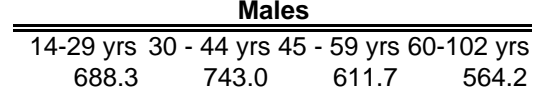

611.7

$\begin{array}{rr}160.0 & (9.1) \\ 64.9 & (13.3) \\ 84.8 & (13.1) \\ 97.6 & (8.3) \\ 25.3 & (4.7)\end{array}$

$\begin{array}{rrrr}169.0 & 169.0 & 165.0 & 161.0 \\ 66.4 & 75.1 & 76.0 & 73.0 \\ 80.5 & 89.4 & 95.0 & 97.7 \\ 93.8 & 98.0 & 98.9 & 99.0 \\ 23.1 & 26.1 & 27.8 & 28.2\end{array}$

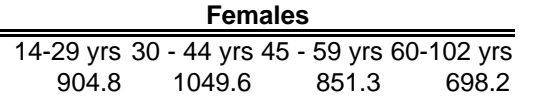

Significant Variation
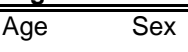

\begin{tabular}{|c|c|c|c|c|c|}
\hline 158.0 & 157.0 & 153.0 & 150.0 & 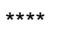 & 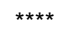 \\
\hline 53.4 & 57.8 & 63.2 & 64.0 & 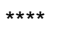 & 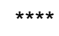 \\
\hline 72.2 & 77.1 & 84.9 & 92.0 & 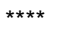 & 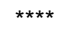 \\
\hline 93.3 & 96.3 & 101.0 & 102.0 & 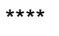 & * \\
\hline 21.3 & 23.5 & 26.8 & 28.5 & 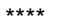 & 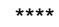 \\
\hline
\end{tabular}

$\begin{array}{rrrr}121.0 & 125.0 & 135.0 & 144 . \\ 71.0 & 79.2 & 84.9 & 83 . \\ 64.8 & 63.8 & 65.7 & 64.2 \\ 6.0 & 6.2 & 6.3 & 6.6 \\ 5.3 & 5.6 & 5.8 & 6.2 \\ 0.5 & 0.5 & 0.6 & 0.7 \\ 513.0 & 623.0 & 741.0 & 925.0\end{array}$

144.0

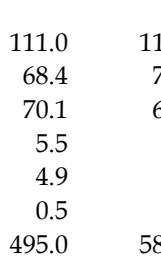

114.0
72.8
68.7
5.5
5.1
0.5
589.0

\begin{tabular}{|c|c|c|}
\hline 129.0 & 140.0 & 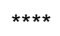 \\
\hline 80.0 & 81.7 & 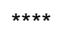 \\
\hline 68.5 & 68.8 & * \\
\hline 5.7 & 6.2 & 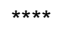 \\
\hline 5.2 & 5.7 & 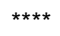 \\
\hline 0.6 & 0.7 & 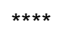 \\
\hline
\end{tabular}

$0.6 \quad(0.1)$

$671.0 \quad(226.0)$

925.0

$48.6 \quad(13.0)$

$93.2 \quad(12.5)$

$\begin{array}{ll}0.2 & (0.1)\end{array}$

$11.0 \quad(3.5)$

$7.2 \quad(2.1)$

49.8

49.8

$\begin{array}{lll}45.9 & 50.1 & 59.9\end{array}$

94.5

102.0

104.0

0.2

16.4
8.9

$43.0 \quad 41.5$

$82.7 \quad 86.7$

$\begin{array}{ll}0.2 & 0.2 \\ 8.4 & 9.3\end{array}$

6.0

9.3
6.8

\begin{tabular}{|c|c|c|c|}
\hline 48.6 & 57.8 & 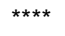 & $\star \star$ \\
\hline 96.2 & 101.0 & $\star \star \star \star *$ & $* *$ \\
\hline 0.2 & 0.2 & 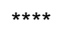 & *夫 \\
\hline 10.8 & 14.1 & 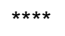 & ** \\
\hline 7.5 & 9.2 & 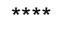 & \\
\hline
\end{tabular}

$38.9 \quad(2.8)$

$15.5 \quad(2.9)$

37.5

37.9

$38.7 \quad 39.4$

$97.8 \quad(27.9)$

$\begin{array}{rr}24.6 & (6.4) \\ 1.9 & (0.5)\end{array}$

$4.1 \quad(1.1)$

$73.7 \quad(26.0)$

\section{Psychological Traits}

NEO N NEUROTICISM

$107.0 \quad(16.2)$

NEO O OPENNESS TO EXPERIENCE $\quad 104.0 \quad(17.4)$

NEO A AGREEABLENESS

$119.0 \quad(15.2)$

$\begin{array}{rrrr}137.0 & 106.0 & 88.5 & 75.1 \\ 28.5 & 25.6 & 23.4 & 18.3 \\ 2.5 & 2.2 & 1.9 & 2.0 \\ 4.9 & 4.3 & 3.9 & 4.3 \\ 73.6 & 70.7 & 70.2 & 79.4 \\ 43.6 & 37.6 & 34.1 & 29.3\end{array}$

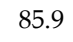

114.0

106.0

108.0

$\begin{array}{rrr}82.4 & 85.5 & 82.6 \\ 111.0 & 106.0 & 103.0 \\ 104.0 & 99.4 & 93.0\end{array}$

$\begin{array}{rrr}114.0 & 117.0 & 124.0\end{array}$
38.6

$$
14.5
$$

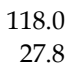

118.0
27.8

2.1

2.1
4.4
68.6

38.9

39.0

15.0

98.2

26.7
1.8

3.8

67.0

39.5

15.4

82.1
24.5
1.6
3.5
74.4
34.3

69.1

18.7

1.7
3.9

3.9
91.6

28.4

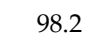

113.0

113.0

116.0
115.0

93.2
107.0
109.0
122.0

93.7
102.0
102.0
124.0

$\begin{array}{rll}94.3 & \star \star \star \star & \star \star \star \star \\ 99.1 & \star \star \star \star & \star \star \star \star \\ 92.9 & \star \star \star \star & \star \star \star \star \\ 129.0 & \star \star \star \star & \star \star \star \star\end{array}$


